\title{
Diseño de un Programa General de Recreación para los estudiantes de la Universidad de Matanzas
}

Recreational Global Program Design for Matanzas University 'students

\author{
Luis Ernesto La O Ramírez. ${ }^{1}$, Tanyara Sánchez Jorge. ${ }^{2}$, Sandra Silvia Santoyo \\ Sánchez. ${ }^{3}$, Lázaro Julio Revoredo Roque. ${ }^{4}$ \& Luis Efraín Velastegui López. ${ }^{5}$
}

\begin{abstract}
.
DOI: https://doi.org/10.33262/concienciadigital.v4i2.1.1715

The research has as main goal a Recreation Global Program design addressed to Matanzas University 'students. In addition, it makes a referential theorist frame about the following topics such as leisure, recreation and touristic animation. It shows a methodological procedure that contributes to solve the main problem of the investigation, inside this proposal is the design and application of a poll for compiling information about the variable academic students' recreational preferences. From the prosecution of the data with the statistical program SPSS (Statistical Product and Service Solutions), it was demonstrated the possibilities that have the students in their free time and the recreational activities that it should plan. Thus, it obtained the best ways to communicate to the students about these recreational activities such as the murals and posters, as well as the student ads. It created a Recreation General Program for each academic headquarters, which was made with a schedule for each single day of the week and an order of activities that consider the weekly frequency of these. It also gives indications for the efficient application of the general program. The methods, techniques and tools that are used: the

\footnotetext{
${ }^{1}$ Universidad de Matanzas, Carrera de Licenciatura en Turismo, Facultad de Ciencias Empresariales. Matanzas, Cuba. Email: luis.lao@umcc.cu

2 Universidad de Matanzas, Carrera de Licenciatura en Turismo, Facultad de Ciencias Empresariales. Matanzas, Cuba. Email: tanyara.sanchez@umcc.cu

${ }^{3}$ Universidad de Matanzas, Carrera de Licenciatura en Turismo, Facultad de Ciencias Empresariales. Matanzas, Cuba. Email: sandra.santoyo@umcc.cu

${ }^{4}$ Hotel Sunbeach, Departamento de Comercial, Carrera de Licenciatura en Turismo. Cárdenas, Cuba. Email: comercial@ sunbeach.gca.tur.cu

${ }^{5}$ Ciencia Digital Editorial, Ecuador, luisefrainvelastegui@cienciadigital.org
} 
historical-logical analysis, the synthesis analysis, the interview, the documentary review, the poll, the brainstorming and the method of experts.

Keywords: leisure, recreation, touristic animation.

\section{Resumen.}

La investigación tiene como objetivo general diseñar un Programa General de Recreación para los estudiantes de la Universidad de Matanzas. Además, se pretende confeccionar un marco teórico referencial sobre los siguientes temas: ocio, recreación y animación turística. También se propone un procedimiento metodológico que contribuye a solucionar el problema general de la investigación. Dentro de esta propuesta se encuentra el diseño y aplicación de una encuesta para recopilar información acerca de la variable preferencias recreativas de los estudiantes universitarios. A partir del procesamiento de los datos con el programa estadístico SPSS (Statistical Product and Service Solutions), se conocerán las posibilidades que tienen los encuestados en su tiempo libre y las actividades recreativas que se deben planificar. Así, se obtuvieron las mejores vías para informar a los estudiantes de las actividades recreativas: los murales y carteles, así como los avisos estudiantiles. Se conformó un Programa General de Recreación para cada sede universitaria, el cual se hizo con un horario para cada uno de los días de la semana y un orden de actividades que toma en cuenta la frecuencia semanal de estas. Asimismo, se ofrecen indicaciones para que la aplicación de dicho programa sea eficiente. Los métodos, técnicas y herramientas utilizadas fueron los siguientes: el análisis histórico-lógico, el análisis síntesis, la entrevista, la revisión documental, la encuesta, la tormenta de ideas y el método de expertos.

Palabras claves: ocio, recreación, animación turística.

\section{Introducción.}

El mundo de hoy tiene infinitas posibilidades de recreación, ya que el recreo es lo que se hace por el placer que se encuentra en ello, sin otra recompensa que la pura actividad; no contribuyendo con él a ganarse la vida. La recreación consiste en encontrar los momentos de diversión durante el uso del tiempo libre y estos deben estar unidos a la relajación y el entretenimiento. Son actividades libres, espontáneas y naturales.

Se caracteriza por ser universal, realizarse en el tiempo libre y por producir satisfacción y agrado. Ofrece oportunidades para el descanso y compensación, de creación y expresión. Involucra actividades auto-motivadas y voluntarias (...) es constructiva y benéfica para el individuo y la sociedad. (Vergara Riveros, 2017) Son ejemplos de ello en todas las regiones geográficas el disfrute de los partidos de futbol en las mejores ligas del mundo, la práctica de los deportes, la participación en los conciertos de los más influyentes grupos musicales, la visualización de los más diversos audiovisuales, la lectura de obras literarias y la conexión a las redes sociales; puesto que la recreación mantiene el equilibrio entre la rutina diaria y las actividades placenteras, evita la muerte prematura de la juventud, enriquece la vida de la gente, contribuye a la dicha humana, al 
desarrollo y bienestar físico, es disciplina, identidad y expresión (...) Fomenta cualidades cívicas, previene la delincuencia y educa a la sociedad para el buen uso del tiempo libre. (Charchabal-Pérez, Luis R. Macao, \& Valverde-Sinche, 2018)

El ocio surge cuando se realizan las actividades satisfactorias y gratificantes que posibilita el tiempo liberado, de forma libre, decididas por uno mismo y gestionadas autónomamente. (Cuenca, 2000) El uso más habitual del concepto está vinculado al descanso del trabajo. El ocio, por lo tanto, aparece fuera del horario laboral o en el periodo de vacaciones. Es importante darle un sentido al ocio (por ejemplo, a través de la práctica de algún deporte, la lectura o la realización de paseos), de lo contrario es probable que este tiempo libre se transforme en aburrimiento. (Sánchez, Gonzalo, \& Miguel Vigil, 2013)

Una de las características relevantes del ocio es la toma de conciencia de lo que se desea hacer en el tiempo libre, lo que implica un adecuado conocimiento de uno mismo y el desarrollo de procesos cognitivos para identificar las actividades que mayor satisfacción pueden generar. Este planteamiento del ocio diferenciado del tiempo libre, lleva directamente hacia planteamientos educativos que generen procesos de identificación y desarrollen la capacidad de elección entre múltiples alternativas. (Chávez Cevallos \& C., 2014)

La animación es un conjunto de técnicas derivadas de la existencia de la recreación como un fenómeno orgánico de carácter social y biológico del hombre, que respaldada por una base científica se organizan y desarrollan en función de satisfacer los intereses de carácter multivariados que presentan los turistas. (Faría, 2008)

Así, la animación turística es gestionar el aprovechamiento del ocio por parte de los clientes, a partir de un sistema de actividades deportivas, lúdicas, festivas, culturales y de aventuras; que muestre lo más auténtico y valedero de nuestra cultura e identidad; que debe ser organizada y planificada de acuerdo al tipo de cliente, sintiendo en todo momento, el cliente, que juega un papel activo en el desarrollo de las actividades.

Si se recorre cada continente se observa en Europa, el disfrute del tenis de campo; en Asia, la afición por el tenis de mesa; en África, la pasión por el baile; en Australia, la tradición por la música, el ballet y el teatro; por último, en América, la tradición por el béisbol y el fútbol en todas sus variantes. De esta manera se llega al continente americano y específicamente a la mayor de las Antillas: Cuba; la cual ha desarrollado una política de recreación sana para su pueblo a partir del triunfo de la Revolución Cubana en enero de 1959.

En el país se divulga todo tipo de diversión vinculada al crecimiento espiritual de sus habitantes y de aquellos que lo visitan, tales son los casos de la Feria Internacional del Libro y la Serie Nacional de Béisbol, dos de las principales opciones de recreación en Cuba. Además, se realizan conciertos de distintos géneros musicales y se disfruta el clima tropical con visitas a las playas y a los ríos del archipiélago. También se tiene una especial 
predilección por juegos de mesa como el dominó. Estos hábitos se han esparcido a las universidades, donde se desarrollan diferentes manifestaciones artísticas a través del Festival de Artistas Aficionados, se saborea el júbilo de la victoria en los eventos deportivos universitarios, se disfruta la música de los compositores que visitan las casas de altos estudios y de todos aquellos creadores artísticos que llevan sus obras a las fiestas estudiantiles.

La recreación para los estudiantes de la Universidad de Matanzas tiene muchos protagonistas, los cuales están clasificados en las distintas áreas que puede abarcar la recreación. Las actividades recreativas en este centro se organizan principalmente por el Departamento de Actividades Extracurriculares y los responsables de esta materia por parte de la Federación Estudiantil Universitaria (FEU). También forman parte de la gestión de actividades recreativas los trabajadores del Departamento de Eventos el cual tiene una programación para favorecer el crecimiento cultural de la comunidad universitaria. En cada facultad se estudian las necesidades recreativas del estudiantado, y la Facultad de Cultura Física ha creado dos planes de actividades físico-recreativas: uno para la Residencia Estudiantil y otro para todos los estudiantes de la Universidad.

Las actividades recreativas son disímiles; por ejemplo, el Festival Universitario del Libro y la Lectura (FULL); el Festival de Artistas Aficionados; se proyectan películas en el Cineclub, viene a la universidad todos los años algún grupo musical y en los laboratorios de informática junto con las zonas Wi Fi del centro, los estudiantes se conectan a Internet.

Actualmente no existe un plan general para la recreación ni en el presupuesto hay una parte especial para dicho tema por lo que en la presente investigación se propone un diseño de un Programa General de Recreación para los estudiantes de la Universidad de Matanzas a partir del análisis de antecedentes y procedimientos de programas de otras universidades tales como Cambridge, Harvard, la Universidad Nacional Autónoma de México (UNAM) y Universidad de Sancti Spíritus "José Martí "; empleando métodos teóricos como el análisis histórico-lógico y el análisis síntesis, métodos empíricos como la entrevista, la revisión documental y la encuesta.

\section{Metodología.}

El aglutinante y el medio para arrojar resultados es confeccionar y aplicar una encuesta. La ruta se dividirá en tres fases, la primera fase abarcará desde la búsqueda y selección de información hasta el diagnóstico de la situación actual en la universidad, la segunda fase comprende la construcción y aplicación de instrumentos para determinar necesidades del estudiantado y la tercera fase de la ruta está marcada por la propuesta de un programa general de recreación, según tabla 1. 


\begin{tabular}{|c|c|c|c|}
\hline Fases & Procedimiento Metodológico & $\begin{array}{l}\text { Métodos, Técnicas } \\
\text { Herramientas }\end{array}$ & y \\
\hline $\begin{array}{l}\text { Diagnóstico de la situación } \\
\text { actual }\end{array}$ & $\begin{array}{l}\text { 1. Identificación de las principales } \\
\text { ofertas recreativas existentes en } \\
\text { la universidad }\end{array}$ & $\begin{array}{l}\text {-Revisión documental } \\
\text {-Entrevistas a estudiantes } \\
\text { personal de la universidad }\end{array}$ & y \\
\hline $\begin{array}{l}\text { Construcción y aplicación de } \\
\text { instrumentos para determinar } \\
\text { necesidades del estudiantado }\end{array}$ & $\begin{array}{l}\text { 2. Diseño de la encuesta a aplicar } \\
\text { 3. Aplicación de encuestas }\end{array}$ & $\begin{array}{l}\text {-Revisión documental } \\
\text {-Tormenta de Ideas } \\
\text {-Método de expertos } \\
\text {-Trabajo de campo } \\
\text {-SPSS }\end{array}$ & \\
\hline $\begin{array}{l}\text { Propuesta de un Programa } \\
\text { General de Recreación }\end{array}$ & $\begin{array}{l}\text { 4. Generalidades } \\
\text { 5. Fundamentación } \\
\text { 6. Objetivos } \\
\text { 7. Metas } \\
\text { 8. Estrategias } \\
\text { 9. Recursos } \\
\text { 10. Análisis de factibilidad } \\
\text { 11. Calendario }\end{array}$ & $\begin{array}{l}\text {-Entrevistas } \\
\text {-Encuestas } \\
\text {-Revisión documental }\end{array}$ & \\
\hline
\end{tabular}

Fuente: Elaboración propia

Fase I: Diagnóstico de la situación actual e identificación de las principales ofertas recreativas existentes en la universidad

Se investigó sobre el estado en el que se encontraba la recreación en la universidad. Se indagó sobre las ofertas recreativas existentes y sobre los responsables de llevarlas a cabo, esto se logró a través de entrevistas a algunos directivos del centro, los cuales están relacionados con el tema y entregaron documentos de gran valor para la investigación.

La revisión documental es una técnica de observación complementaria, en caso de que exista registro de acciones y programas. La revisión documental permite hacerse una idea del desarrollo y las características de los procesos y también de disponer de información que confirme o haga dudar de lo que el grupo entrevistado ha mencionado.

Una entrevista es un intercambio de ideas, opiniones mediante una conversación que se da entre una, dos o más personas donde un entrevistador es el designado para preguntar. Es recíproca, donde el entrevistado utiliza una técnica de recolección mediante una interrogación estructurada o una conversación totalmente libre; en ambos casos se utiliza un formulario o esquema con preguntas o cuestiones para enfocar la charla, que sirven como guía. (Poll Gutiérrez, 2019)

Fase II: Construcción y aplicación de instrumentos para determinar necesidades de los estudiantes. Diseño de encuestas a aplicar.

La encuesta posee al inicio una parte para conocer los datos socio-demográficos de los encuestados (sexo, edad, año que cursa, si posee beca y la carrera que estudia). El test posee 9 preguntas, la primera estudia la cantidad de horas libres que tienen los estudiantes universitarios por cada día de la semana; la segunda persigue conseguir una valoración del tiempo libre para realizar las actividades recreativas, donde se puede dar el caso que marque "Poco" y esto llevaría al encuestado a expresar los factores que limitan ese tiempo. La tercera trata sobre las vías de información de los estudiantes, sobre su asistencia a las actividades recreativas y las causas que justifican lo que marcaron en cuanto a la asistencia; la cuarta quiere encontrar el orden de preferencia de los estudiantes en cuanto a las actividades que pudieran realizar en dos momentos del día: después del 
horario docente y después de la comida; la quinta busca consideraciones acerca de la programación de actividades en el campismo; la sexta desea ordenar según las preferencias aquellas actividades recreativas que ofrece la Universidad de Matanzas; la séptima trata sobre la sistematicidad de las actividades que oferta la Universidad de Matanzas; la octava le da la posibilidad al encuestado de proponer nuevas actividades recreativas y la novena tiene como objetivo seleccionar los grupos musicales que realizarán conciertos cada año en el centro. Todas las preguntas son cerradas; pero la 2 , la 3 , la 4 , la 6 , la 7 , la 8 y la 9 dan la posibilidad de incrementar las variantes en cada pregunta o de añadir propuestas a través de las pequeñas tablas que se agregan al final de cada una de ellas.

El universo y muestra a encuestar estuvo constituido por 3035 adolescentes y jóvenes que estudiaban en el municipio de Matanzas durante el curso 2018-2019. Se excluyeron los estudiantes que no fueron seleccionados aleatoriamente o los que no se encontraban en el centro, los que no presentaron disposición a participar y los que no eran del Curso Regular Diurno. Se determinó el tamaño de la muestra a partir de la siguiente expresión (Figura

$$
n=\frac{N^{*} Z_{1-\alpha}{ }^{2}{ }^{*} p^{*} q}{d^{2} *(N-1)+Z_{1-\alpha}{ }^{2} * p^{*} q}
$$

\section{Ecuación 1: cálculo de} muestra

1):

Siendo:

p: Prevalencia de éxito.

q: Complemento de $\mathrm{p}$.

d: Precisión.

$\mathrm{n}$ : tamaño de la muestra

$\mathrm{N}$ : tamaño de la población

$\alpha$ : Error alfa.

Z (1- $\alpha$ ): Nivel de confianza.

1- $\alpha$ : Confiabilidad.

Se utilizó una p de 50\%, se fijó un valor máximo de precisión d = 5\% y la confiabilidad que se usó es de 95\%. Se calculó el tamaño de la muestra para un nivel de confianza de 2 y se obtuvo un tamaño mínimo necesario de aproximadamente 354 estudiantes, luego se calculó el tamaño de la muestra para un nivel de confianza de 1,96 y se obtuvo un tamaño mínimo necesario de aproximadamente 342 estudiantes; esto trajo como consecuencia que se realizaran 353 encuestas válidas. En la selección de los sujetos se utilizó un muestreo estratificado proporcional con la utilización de un generador de números aleatorios.

Se calcularon tamaños de muestras proporcionales a la población total de cada estrato, para lo cual se empleó la fórmula descrita a continuación (Figura 2):

$$
\frac{x}{T}=\frac{n}{N} \quad \begin{aligned}
& \text { Figura 2: Ecuación para el cálculo de } \\
& \text { tamaño de muestra proporcional al total. }
\end{aligned}
$$

X: cantidad de estudiantes a encuestar en cada estrato.

T: cantidad total de estudiantes en cada estrato. 
n: tamaño de la muestra.

$\mathrm{N}$ : tamaño de la población.

La muestra quedó distribuida de la siguiente forma (Tabla 2):

Tabla 2. Distribución de la muestra.

\begin{tabular}{lcc}
\hline Estratos & Cantidad total de estudiantes & Tamaño de la muestra \\
\hline Sede Camilo Cienfuegos & 2597 & 302 \\
\hline Sede Juan Marinello & 438 & 51 \\
\hline Total & 3035 & 342
\end{tabular}

Fuente: Elaboración propia

El universo de expertos estuvo conformado por profesionales con competencia demostrada y experiencia documentada. A partir de un muestreo intencional se seleccionan 7 personas con experiencia laboral en el área de recreación y cultura, con más de cinco años en alguna de las siguientes áreas: recreación, ocio, cultura física y animación, o con contribución científica (artículos, tesis y libros) relacionada con el tema y que aceptaron participar en el estudio. Estos expertos contribuyeron con la validación de la encuesta.

La aplicación de encuestas es el último paso después de haber cumplido otros que le aportarían lo necesario para realizarse. para seleccionar a los encuestados se necesitó hacer dos listas enumeradas con los estudiantes de cada sede universitaria (Sede Camilo Cienfuegos y Sede Juan Marinello), una lista iba del 1 al 2597 y la otra del 1 al 438; así se generaron números aleatorios con un generador de estos, para cada estrato, y cada número aleatorio generado correspondía a un estudiante; se generaron tantos números como indicaba el tamaño de la muestra en cada estrato.

La selección de los encuestados nos lleva a realizar una prueba piloto a 10 de ellos. El objetivo de esta fue definir si existían dificultades en la comprensión, claridad, diseño, formato, sentimientos que les produjo y recomendaciones, para poder adaptar el test al lenguaje y nivel cultural de los jóvenes a los que iba dirigido el estudio. Después de aplicada la prueba piloto, comenzó la aplicación formal de la encuesta.

El SPSS es un software conocido y utilizado para la realización de investigaciones de mercado e investigaciones relacionadas al área de la sociología y psicología. Una de las características fundamentales del SPSS es su facilidad de uso, junto a la potencia e integridad del software, convirtiéndolo en una de las herramientas más potentes para éste tipo de trabajos.

Fase III: Propuesta de un programa general de Recreación

-Generalidades del programa: El programa recreativo tiene una serie de actividades recreativas y deportivas que tienden a satisfacer la amplia diversidad de intereses de los estudiantes, y a su vez existen las condiciones mínimas indispensables para llevarlas a cabo. 
-Fundamentación del programa: se realizó un diagnóstico de la situación actual de la recreación en la Universidad de Matanzas y esto corroboró que no existía un Programa General de Recreación, el cual debía agrupar todas las actividades recreativas ofrecidas por el centro. De esta forma, se pasó a realizar una encuesta que permitiera agrupar toda la información necesaria para elaborar ese programa, dicho instrumento midió la variable preferencias recreativas de los estudiantes universitarios.

-Objetivos: organizar un cúmulo de actividades que son de interés de los estudiantes de la Universidad de Matanzas, contribuir al desarrollo sistemático de actividades recreativas y aprovechamiento del tiempo libre de los estudiantes, facilitar a través de estas actividades la adquisición de conocimientos en los estudiantes, hacer más placentera la estancia de los estudiantes en el centro durante el curso.

-Meta: lograr la mayor participación de los estudiantes en las actividades programadas por la escuela hasta llegar a la incorporación masiva del estudiantado universitario.

-Estrategias: Realizar una divulgación del mismo a través de anuncios, pancartas, radio base y la brigada de la FEU durante todo el curso. Capacitar a los diferentes profesores para que puedan enfrentar con éxito el desarrollo del programa. Desarrollar una estrecha coordinación con instituciones y organismos cercanos para poder hacer más amplia y variada la cantidad de actividades a desarrollar. Utilizar con eficacia y eficiencia el conjunto de los recursos humanos y materiales que determinan la calidad de las actividades.

-Recursos: Para la inclusión e incursión de las actividades del programa se tuvo en cuenta tanto los recursos humanos como materiales existentes en el centro (Tabla 3):

Tabla 3. Recursos para las actividades de los programas.

\begin{tabular}{ll}
\hline Recursos humanos & Recursos materiales \\
\hline -Profesores. & -Implementos deportivos. \\
\hline -Personal de apoyo administrativo. & -Instalaciones deportivas. \\
\hline -Personal de dirección. & -Instalaciones culturales y recreativas. \\
\hline -Consejo de la FEU. & -Áreas de juegos pasivos (ludoteca). \\
\hline -Dirección de la UJC. & -Bases de campismos. \\
\hline -Consejo de residencia estudiantil. & -Biblioteca. \\
\hline -Dirección de Extensión Universitaria. & \\
\hline -Personal de medios de propaganda. & \\
\hline
\end{tabular}

Fuente: Tomado de (Luna Pino, Piña León, Bravo Rojas, \& Caridad Dorta Martínez, 2000)

-Análisis de factibilidad: para la realización del programa no son necesarias grandes inversiones, sólo el uso adecuado y racional de los recursos que se disponen, así como los medios tanto deportivos como culturales y el establecimiento de una coordinación por parte de la dirección de la UJC y la FEU del centro con el Campismo Popular, para proponer como oferta sus diferentes bases. 
-Calendario: se pondrá en práctica en las dos sedes universitarias de la Universidad de Matanzas a partir del curso 2021-2022 y se le podrá hacer cambios significativos un año después de su puesta en marcha.

\section{Resultados.}

La Universidad de Matanzas es una destacada institución universitaria que ha experimentado desde sus inicios un vertiginoso y potente desarrollo educacional, que llega a ser reconocida como una de las cinco mejores universidades cubanas, según el Ministerio de Educación Superior (MES). Sus orígenes se remontan al 9 de mayo de 1972, cuando con apoyo de la Universidad de La Habana y del Comité Provincial del Partido Comunista de Cuba se creó la Sede Universitaria de Matanzas, que fungiría como filial de la primera, con la finalidad de atender a todas las carreras y cursos de nivel superior que se ofrecían en la provincia. Se instauraba así la Educación Superior en la provincia. La sede se desarrolló vertiginosamente hasta convertirse, en 1976, en tres centros: el Instituto Pedagógico, la Facultad de Medicina y el Centro Universitario (la futura Universidad de Matanzas). La sede fue creada sin contar con los recursos técnicos y materiales, sin tener todas las condiciones requeridas para el desarrollo normal de un centro universitario.

Las Fases I y II (Diagnóstico de la situación actual; Construcción y aplicación de instrumentos para determinar necesidades del estudiantado) tiene desarrollo en las etapas 1; 2 y 3 con el empleo de métodos, técnicas y herramientas de análisis de datos recopilados y procesados en Microsoft Excel y SPSS.

Datos Sociodemográficos, relación entre Año que cursa y Estado Becario del estudiante por Especialidades (Sede Universitaria Camilo Cienfuegos): Con un total de encuestados de 302 estudiantes, el $56.29 \%$ son del género masculino y $43.71 \%$ son del género femenino; el rango de edad va desde los 18 hasta los 28 años, donde la mayor masa de encuestados fue de 21 años; el $72 \%$ son estudiantes externos. La muestra tomó estudiantes de todos los años, los de mayor presencia fuero los estudiantes de tercer año (3er Año) con un 35, $43 \%$.

Datos Sociodemográficos, relación entre Sexo y Edad por Especialidades (Sede Juan Marinello): Con un total de encuestados de 51 estudiantes, el 80,39\% son del género femenino y $19.61 \%$ son del género masculino; el rango de edad va desde los 18 hasta los 25 años, donde la mayor masa de encuestados fue de 21 años; el $60.78 \%$ son estudiantes externos. La muestra tomó estudiantes de todos los años, los de mayor presencia fueron los estudiantes de tercer año (3er Año) con un 25,49\%.

Cantidad de tiempo libre que manifiestan tener los estudiantes becados y externos de las sedes Camilos Cienfuegos y Juan Marinello.

Para la sede Camilo Cienfuegos, la mayor marcación en la relación de menos tiempo libre está en el lunes, con 112 marcaciones, con un peso del 70,53\% en los estudiantes 
externos. La mayor marcación en la relación de más tiempo libre está en los sábados, con un peso del $74 \%$ en los estudiantes externos (tabla 4).

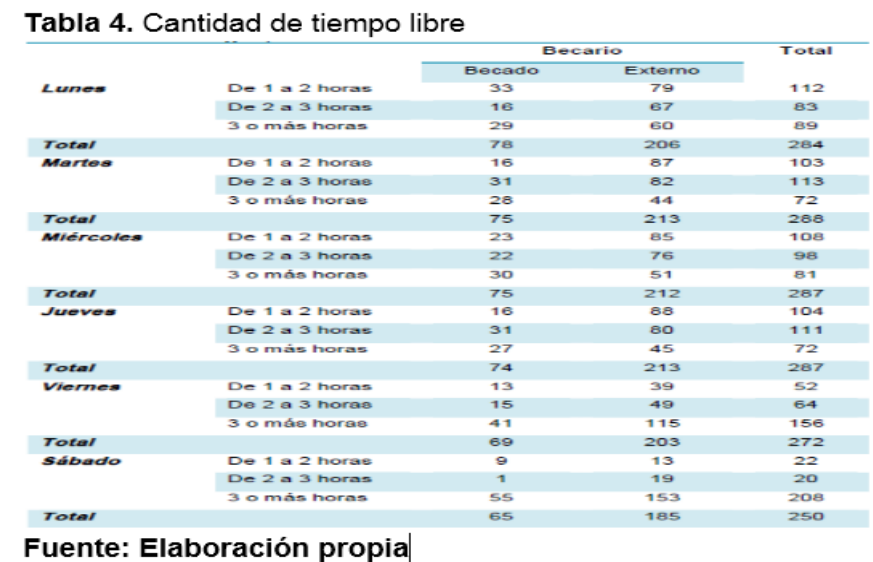

En la sede Juan Marinello la mayor marcación en la relación de menos tiempo libre está en el jueves, con 9 marcaciones, y con un peso del $77.7 \%$ en los estudiantes externos. La mayor marcación en la relación de más tiempo libre está en los sábados, con un peso del $65.12 \%$ en los estudiantes externos (tabla 5).

Tabla 5. Tiempo libre.

\begin{tabular}{|c|c|c|c|c|}
\hline & & \multicolumn{2}{|c|}{ Becado } & \multirow[t]{2}{*}{ Total } \\
\hline & & Becado & Externo & \\
\hline \multirow[t]{3}{*}{ Lunes } & De 1 a 2 horas & 1 & 7 & 8 \\
\hline & $\mathrm{De} 2$ a 3 horas & 8 & 7 & 15 \\
\hline & 30 más horas & 10 & 15 & 25 \\
\hline \multirow[t]{3}{*}{ Martes } & De 1 a 2 horas & 2 & 5 & 7 \\
\hline & De 2 a 3 horas & 4 & 9 & 13 \\
\hline & 30 más horas & 13 & 15 & 28 \\
\hline \multirow[t]{3}{*}{ Miércoles } & De 1 a 2 horas & 0 & 7 & 7 \\
\hline & De 2 a 3 horas & 6 & 7 & 13 \\
\hline & 30 más horas & 14 & 15 & 29 \\
\hline \multirow[t]{3}{*}{ Jueves } & De 1 a 2 horas & 2 & 7 & 9 \\
\hline & De 2 a 3 horas & 7 & 7 & 14 \\
\hline & 3 o más horas & 11 & 15 & 26 \\
\hline \multirow[t]{4}{*}{ Viernes } & & 1 & 2 & 3 \\
\hline & De 1 a 2 horas & 0 & 4 & 4 \\
\hline & De 2 a 3 horas & 5 & 5 & 10 \\
\hline & 30 más horas & 14 & 20 & 34 \\
\hline \multirow[t]{3}{*}{ Sábado } & De 1 a 2 horas & 1 & 1 & 2 \\
\hline & De 2 a 3 horas & 0 & 3 & 3 \\
\hline & 30 más horas & 14 & 24 & 38 \\
\hline
\end{tabular}

Fuente: Elaboración propia

Comportamiento de uso y efectividad de los medios de comunicación empleados para dar a conocer las actividades planificadas por el centro.

En la sede Camilo Cienfuegos el medio más efectivo es el empleo de murales y carteles con 186 marcaciones y los más enterados por subtotal o por ciento de éxito son los becados con un $63 \%$ ante un $61 \%$ los externos (tabla 6 ). 
Tabla 6. Comportamiento de uso y efectividad de los medios de comunicación (Camilo Cienfuegos).

\begin{tabular}{lccccc}
\hline Becario & $\begin{array}{c}\text { Radio } \\
\text { Base }\end{array}$ & $\begin{array}{c}\text { Murales } \boldsymbol{y} \\
\text { carteles }\end{array}$ & $\begin{array}{c}\text { No tengo } \\
\text { información }\end{array}$ & $\begin{array}{c}\text { Correos } \\
\text { electrónicos }\end{array}$ & $\begin{array}{c}\text { Avisos } \\
\text { estudiantiles }\end{array}$ \\
\hline Becado & 0 & 53 & 11 & 4 & 51 \\
Externo & 0 & 133 & 27 & 4 & 125 \\
Totales & 0 & 186 & 38 & 8 & 176 \\
\hline
\end{tabular}

Fuente: Elaboración propia

En la sede Juan Marinello el medio más efectivo es el empleo de avisos estudiantiles con 45 marcaciones y los más enterados por subtotal o por ciento de éxito son los becados con un $90 \%$ ante un $87 \%$ los externos (tabla 7).

Tabla 7. Comportamiento de uso y efectividad de los medios de comunicación (Juan

\begin{tabular}{lccccc} 
Becario & $\begin{array}{c}\text { Radio } \\
\text { Base }\end{array}$ & $\begin{array}{c}\text { Murales } \boldsymbol{y} \\
\text { carteles }\end{array}$ & $\begin{array}{c}\text { No tengo } \\
\text { información }\end{array}$ & $\begin{array}{c}\text { Correos } \\
\text { electrónicos }\end{array}$ & $\begin{array}{c}\text { Avisos } \\
\text { estudiantiles }\end{array}$ \\
\hline Becado & 0 & 8 & 1 & 3 & 18 \\
Externo & 0 & 9 & 3 & 1 & 27 \\
Totales & 0 & 17 & 4 & 4 & 45 \\
\hline
\end{tabular}

Fuente: Elaboración propia

\section{Asistencia estudiantil a las actividades planificadas por el centro.}

La sede Camilo Cienfuegos es la más efectiva en cuanto al nivel de participación de estudiantes en las actividades que se planifican y se divulgan, con un $10 \%$ de estudiantes que siempre van a las actividades (Tabla 8).

Tabla 8: Nivel de participación (Camilo Cienfuegos)

\begin{tabular}{lcccc}
\hline Becario & Nunca & A veces & Siempre & \\
\hline Becado & 1 & 66 & 17 & 84 \\
Externo & 16 & 186 & 16 & 218 \\
Totales & 17 & 252 & 33 & 302 \\
\hline
\end{tabular}

Fuente: Elaboración propia.

La Juan Marinello cuenta con un $2 \%$ que siempre van, "pero es superior a la Camilo en masa de estudiantes que a veces participan en las actividades con un $94.11 \%$ ante un 83 $\%$. En ambas sedes la mayor cifra de los que siempre van está en los becarios internos y de los que a veces van está en los externos (tabla 9).

\begin{tabular}{ccccc}
\multicolumn{5}{c}{ Tabla 9: Nivel de participación (Juan Marinello) } \\
\hline Becario & Nunca & A veces & Siempre & \\
\hline Becado & 1 & 18 & 1 & 20 \\
Externo & 1 & 30 & 0 & 31 \\
Totales & 2 & 48 & 1 & 51 \\
\hline
\end{tabular}

Fuente: Elaboración propia. 
Preferencias de actividades después del horario docente y después de comida (Sede Universitaria Camilo Cienfuegos): La actividad de mayor marcación una vez culminado el tiempo docente fue: "Conversar con amigos"; la actividad más marcada en 1era opción es "Retirarse hacia la casa". La actividad de mayor frecuencia de marcación entre las tres primeras opciones después del horario docente: "Conversar con amigos". Además de las actividades plasmadas en la encuesta, los estudiantes manifestaron hacer otras actividades que son de su preferencia: jugar con el teléfono, ir para casa de amigos, ir a la playa, caminar con mi familia, ir a la playa, pasar tiempo con mi pareja, actividades festivas, presentación de grupos musicales, presentación de obras de teatro, leer, ejercicios aeróbicos, dormir, entre otras. La actividad de mayor marcación en el horario posterior a la comida es: "Conexión a Internet"; la actividad más marcada en 1era opción es "Descanso pasivo". La actividad de mayor frecuencia de marcación entre las tres primeras opciones después de comida es: "Conexión a internet".

Preferencias de actividades después del horario docente y después de comida (Sede Juan Marinello): La actividad de mayor marcación una vez culminado el horario dicente fue: "Conversar con amigos"; la actividad más marcada en lera opción es "Retirarse hacia la casa". La actividad de mayor frecuencia de marcación entre las tres primeras opciones después del horario docente: "Descanso pasivo". La actividad de mayor marcación en el horario posterior a la comida es: "Visitar centros nocturnos"; la actividad más marcada en lera opción es "Descanso pasivo". La actividad de mayor frecuencia de marcación entre las tres primeras opciones después de comida es: "Conexión a Internet".

Tabla 10. Análisis en el orden de preferencia estudiantil de las actividades ofrecidas y planificadas por la Sede Universitaria Camilo Cienfuegos

\begin{tabular}{|c|c|c|c|c|c|c|}
\hline No. & Actividades según intereses & Conteo & $\begin{array}{c}\text { 1era } \\
\text { opción }\end{array}$ & $\begin{array}{c}\text { 2da } \\
\text { opción }\end{array}$ & $\begin{array}{c}\text { 3era } \\
\text { opción }\end{array}$ & $\begin{array}{c}\text { Frecuencia en las tres } \\
\text { primeras opciones }\end{array}$ \\
\hline 1 & Encuentros deportivos & 270 & 58 & 49 & 43 & 150 \\
\hline 2 & $\begin{array}{l}\text { Conciertos de grupos } \\
\text { nacionales }\end{array}$ & 285 & 133 & 140 & 28 & 301 \\
\hline 3 & Encuentros & 238 & 7 & 28 & 39 & 74 \\
\hline 4 & Música bailable & 265 & 59 & 75 & 32 & 166 \\
\hline 5 & Coloquio de la décima & 232 & 5 & 2 & 14 & 21 \\
\hline 6 & Galería Abierta & 242 & 7 & 12 & 25 & 44 \\
\hline 7 & A ciencia Cierta & 237 & 1 & 7 & 21 & 29 \\
\hline 8 & $\begin{array}{l}\text { Festival universitario del } \\
\text { libro y la literatura }\end{array}$ & 245 & 7 & 12 & 18 & 37 \\
\hline 9 & $\begin{array}{l}\text { Presentación de Proyectos } \\
\text { audiovisuales }\end{array}$ & 248 & 9 & 22 & 43 & 74 \\
\hline 10 & Artes Escénicas & 232 & 7 & 10 & 25 & 42 ctivar Win \\
\hline
\end{tabular}

Fuente: Elaboración propia. 
Tabla 11. Análisis en el orden de preferencia estudiantil de las actividades ofrecidas y planificadas por la Sede Juan Marinello.

\begin{tabular}{|c|c|c|c|c|c|c|}
\hline No. & Actividades según intereses & Conteo & $\begin{array}{c}\text { 1era } \\
\text { opción }\end{array}$ & $\begin{array}{c}\text { 2da } \\
\text { opción }\end{array}$ & $\begin{array}{c}\text { 3era } \\
\text { opción }\end{array}$ & $\begin{array}{c}\text { Frecuencia en las } \\
\text { tres primeras } \\
\text { opciones }\end{array}$ \\
\hline 1 & Encuentros deportivos & 33 & 1 & 2 & 7 & 10 \\
\hline 2 & $\begin{array}{l}\text { Conciertos de grupos } \\
\text { nacionales }\end{array}$ & 47 & 34 & 9 & 1 & 44 \\
\hline 3 & Encuentros & 31 & 3 & 3 & 6 & 12 \\
\hline 4 & Música bailable & 41 & 6 & 15 & 10 & 31 \\
\hline 5 & Coloquio de la décima & 25 & 0 & 1 & 4 & 5 \\
\hline 6 & Galería Abierta & 32 & 1 & 2 & 2 & 5 \\
\hline 7 & A ciencia Cierta & 25 & 2 & 2 & 2 & 6 \\
\hline 8 & $\begin{array}{l}\text { Festival universitario del libro } \\
\text { y la literatura }\end{array}$ & 31 & 1 & 9 & 1 & 11 \\
\hline 9 & $\begin{array}{l}\text { Presentación de proyectos } \\
\text { audiovisuales }\end{array}$ & 41 & 2 & 10 & 9 & 21 \\
\hline 10 & Artes Escénicas & 30 & 0 & 2 & 8 & 10 \\
\hline
\end{tabular}

Fuente: Elaboración propia.

La actividad de mayor marcación en la preferencia de ambas sedes es el Concierto de grupos nacionales; la actividad más marcada en lera opción coincide para las dos sedes y es el concierto de grupos nacionales. La actividad de mayor frecuencia de marcación entre las tres primeras opciones en ambas sedes también son los conciertos de grupos nacionales. En modo de resumen, esta es la actividad preferida y de mayores porcientos de asistencia por parte del estudiantado.

Fase III Programa General de Recreación

El Programa General de Recreación fue confeccionado según los siguientes pasos:

1. Reunir los resultados de las preguntas $4 ; 6 ; 7$ y 8.

2. Analizar la pregunta 4 según los dos momentos del día.

3. A partir de aquí se observaron las tres primeras opciones en la preferencia de los encuestados para ver si se podían insertar en el Programa General de Recreación y se observó que eran actividades que los estudiantes hacían por su cuenta, por ejemplo: conversar con amigos, retirarse hacia la casa y descanso pasivo. Por esta razón, se hizo una lista con todas las actividades que contiene esa pregunta y se escogieron aquellas que tenían mayor preferencia a partir de la suma de las frecuencias en las cuales los estudiantes pusieron las actividades en primera, segunda y tercera opción.

En esta lista se desecharon las tres últimas opciones o todas aquellas que estuvieran empatadas en el último lugar. Entonces se fueron colocando en una tabla que contenía los días de la semana y el horario docente de la universidad, dicho horario llegaba hasta las diez de la noche (se hizo el programa de forma semanal). Se tomó en cuenta que hay dos sesiones a la hora de las clases y que siempre queda un tiempo disponible para hacer lo que las personas estimen conveniente; además, se eligió un local como sede principal de las actividades recreativas, se deja un tiempo para almorzar y para comer, así como para las tareas sanitarias; teniendo en cuenta todo esto, se estimó necesario, que por la importancia del estudio y la repercusión del gasto de energía por parte de los estudiantes, 
se programaran actividades de lunes a viernes dentro de la sede universitaria y se dejaran para el fin de semana aquellas actividades que se realizan fuera del centro.

4. Los resultados de la pregunta 6 sirvieron para elegir actividades que se pondrían por encima de otras en cuanto a su frecuencia semanal, por ejemplo, los encuentros deportivos pueden estar por encima de los proyectos audiovisuales.

5. Los resultados de la pregunta 7 sirvieron para saber las veces que se repetía semanalmente cada actividad recreativa.

6. Los resultados de la pregunta 8 sirvieron para insertar actividades novedosas dentro del programa.

Tabla 12. Programa General de Recreación semanal para la Sede Camilo Cienfuegos.

\begin{tabular}{llll} 
Horario & lunes & martes & miércoles \\
\hline 8:00am-11:20am & Dominó & Ver audiovisuales & Jugar videojuegos \\
\hline 1:30pm-5:00pm & Fútbol & Baloncesto & Voleibol \\
\hline 8:30pm-10:00pm & Ver audiovisuales & Ver audiovisuales & Ver audiovisuales \\
\hline Horario & jueves & viernes & sábado \\
\hline 8:00am-11:20am & Dominó & Dominó & Excursiones \\
\hline 1:30pm-5:00pm & Fútbol & & \\
\hline 8:30pm-10:00pm & Ver audiovisuales & & \\
& & &
\end{tabular}

Fuente: Elaboración propia.

Tabla 13. Programa General de Recreación semanal para la Sede Juan Marinello.

\begin{tabular}{llll}
\hline Horario & lunes & martes & miércoles \\
\hline 8:00am-11:20am & Ver audiovisuales & Ajedrez & Jugar videojuegos \\
\hline 1:30pm-5:00pm & Dominó & Voleibol & Voleibol \\
\hline 8:30pm-10:00pm & Música bailable & Música bailable & Música bailable \\
\hline Horario & jueves & viernes & sábado \\
\hline 8:00am-11:20am & Ver audiovisuales & Ver audiovisuales & Excursiones \\
\cline { 1 - 2 } 1:30pm-5:00pm & Dominó & & \\
\hline 8:30pm-10:00pm & Música bailable & & \\
\hline
\end{tabular}

Fuente: Elaboración propia.

\section{Conclusiones}

- El procedimiento metodológico de la investigación permite hacer un diagnóstico de la situación actual de la recreación en la universidad, recopilar la información necesaria para conocer las preferencias recreativas de los estudiantes y elaborar un Programa General de Recreación que reúna todas las condiciones para ser aplicado satisfactoriamente, todo esto mediante la aplicación de diversas técnicas, métodos y herramientas.

- La propuesta de un Programa General de Recreación contribuirá a la formación 
integral de los estudiantes universitarios permitiendo su integración con la naturaleza, preservando los recursos naturales y haciendo buen uso del tiempo libre, lo cual repercutirá en el orden, la disciplina y la exigencia de la Universidad de Matanzas.

- Con la investigación el estudiante universitario tendrá la oportunidad de realizar actividades que favorezcan su desarrollo, adquirirá destrezas y podrá cooperar como ciudadano en la construcción de una mejor forma de vida para compartirla con toda la comunidad.

- A través de la encuesta aplicada en esta investigación se conocieron las preferencias de los estudiantes universitarios y se demostró a través de los análisis de frecuencia la tendencia hacia el uso de las redes sociales como actividad recreativa.

\section{Referencias bibliográficas}

Acevedo. (2015). Condiciones de la recreación comunitaria a través de sus prácticas, espacios y sujetos en los contextos de la transformación de la ciudad de Medellín. CONNOTA. No Vol. 1.

Aguilar. (2017). Prácticas de ocio y tiempo libre en tres generaciones: un estudio de caso en la Universidad YMCA (México). [Tesis de Tesis doctoral.], en opción al Universidad de Deusto.

Arias, F. J., \& Ed. (2008). Herramientas de Apoyo a la Solución de Problemas no Estructurados en Empresas Turísticas. HASPNET.

Bejarano, C. (2011). Un Saber Hacer para la construcción de un enfoque CTS (Ciencia, Tecnología y Sociedad). Los actores de la Extensión Universitaria. .

Bermúdez-Tirado, S., Ramírez-Hoyos, M., \& Jaramillo-Arias, A. .. (2016). Preferencias en el uso del tiempo libre de los estudiantes universitarios con enfoque en el sector turismo. $C E A$.

Brito Mayea, Y. B., Ferreiro, Y., \& Hernández, Y. (2013). Plan de comunicación para fortalecer el proceso de extensión universitaria en la Universidad de Sancti Spíritus. Márgenes. No (No. 1 ) Vol. 1.

Carazo Vargas, P. C., \& C., K. .. ( 2015). Recreación como estrategia para el afrontamiento del estrés en ambientes laborales. EmásF.

Carmona Lopera, V. (2015). Aprovechamiento del tiempo libre y realización de actividad física regular en comunidad universitaria de Colombia. Prueba piloto.

Castillo Viera, E. a., Guerra, F. J., \& Robles Rodríguez, J. (2012). Diseño de un cuestionario sobre hábitos de actividad física y estilo de vida a partir del método Delphi. E-balonmano.com: Revista de Ciencias del Deporte., 1885 - 7019. 
Castillo Viera, E. G., Francisco Javier, G., \& Sáenz-López Buñuel, P. (2009). Ocupación del tiempo libre del alumnado en la Universidad de Huelva. E-balonmano.com: Revista de Ciencias del Deporte, 1885 - 7019.

Charchabal-Pérez, D. V.-J., Luis R. Macao, K. N., \& Valverde-Sinche, L. V. (2018). La recreación y su impacto en el sedentarismo en los estudiantes universitarios. OLIMPIA. No (Núm. 48: Edición especial) 15., p. 1817-9088.

Chávez Cevallos, E. S., \& C., L. (2014). Nacional Juan de Salinas de Sangolquí Estudio del tiempo libre de los adolescentes ecuatorianos del Colegio. EFDeportes.com. No $\left(N^{o} 188\right)$.

Chávez Cevallos, E., \& S. C., L. (2014). Estudio del tiempo libre de los adolescentes ecuatorianos del Colegio Nacional Juan de Salinas de Sangolquí. EFDeportes.com. No ( $\left.N^{o} 188\right)$.

Cuenca. (2000). Investigación sobre el ocio.

Expósito, J. G., Sanhueza, L. M., Angulo, C., \& Teresa, M. (2008). Análisis de las actividades de ocio en estudiantes de primer curso de la Facultad de Educación: Relación con el alcohol.

Faría, B. (2008). Animación.

Hernández Blanco, A. (2007). Proyecto de Extensión Universitaria para Sede Pedagógica: "Pelayo Villanueva Valverde" del municipio Colón.

León De La Portilla, Y. (2012). Repercusión de las acciones de extensión universitaria, como proceso sustantivo de la educación superior cubana, en los modos de actuación de los egresados de la carrera de Estudios Socioculturales. [Tesis de Tesis en opción al título de Licenciado en Estudios Socioculturales.], en opción al Universidad de Matanzas.

Luce Gomes, C. O., Esperanza, P., \& Leila. Elizalde, R. (2009). Lazer na América Latina /Tiempo Libre Ocio y Recreación en Latinoamérica. Editora UFMG.

Luna Pino, G. C., Piña León, E., Bravo Rojas, P. O., \& Caridad Dorta Martínez, E. (2000). Estudio de las necesidades recreativas de los estudiantes en la Facultad de Ciencias Médicas y su tiempo libre.

Poll Gutiérrez, H. (2019). Proyecto sociocultural "Aya Ata" como instrumento de comunicación en el perfeccionamiento de la animación turística cultural del hotel Muthu Playa Varadero.

Rangel-Lyne, L. O., \& H., M. L. (2012). El tiempo libre de los estudiantes universitarios de la Facultad de Comercio y Administración de Tampico. CienciaUAT. No (3) 6. 
Sánchez, M. G., Gonzalo, E., \& Miguel Vigil, E. (2013). ¿Qué hacemos en nuestro tiempo libre?

Sandoval, N. ( 2017). Diagnóstico acerca del uso del ocio y el tiempo libre entre los estudiantes de la Universidad Nacional Experimental del Táchira. Pedagogía Social. No (30).

Tortosa-Martínez, J. (2011). Las titulaciones de ocio y recreación en los Estados Unidos: un análisis comparativo. Revista de Investigación en Educación. No (9).

Tortosa-Martínez, J. (2012). La recreación como profesión en Estados Unidos. Revista Española de Educación Física y Deportes. No N. ${ }^{\circ} 396$.

Vergara Riveros, F. A. (2017). Una mirada desde la Educación Física a las políticas públicas: el Consejo Local de Deportes y Recreación de la localidad de Kennedy, Bogotá (2006-2016). Colombia: [Tesis de Tesis de grado presentada para optar por el Título de Magíster en Educación.], en opción al Universidad Nacional.

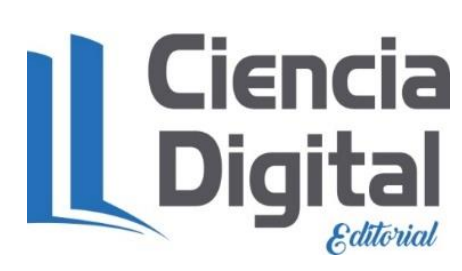




\section{PARA CITAR EL ARTÍCULO INDEXADO.}

La O Ramírez, L. E., Sánchez Jorge, T., Santoyo Sánchez., S. S., Revoredo Roque, L. J., \& Velastegui López, L. E. (2021). Diseño de un Programa General de Recreación para los estudiantes de la Universidad de Matanzas. ConcienciaDigital, 4(2.1), 113-130. https://doi.org/10.33262/concienciadigital.v4i2.1.1715

\section{LCiencia}

El artículo que se publica es de exclusiva responsabilidad de los autores y no necesariamente reflejan el pensamiento de la Revista Conciencia Digital.

El artículo queda en propiedad de la revista y, por tanto, su publicación parcial y/o total en otro medio tiene que ser autorizado por el director de la Revista Conciencia Digital.

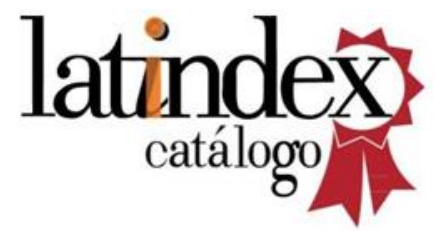

